\title{
Sending Signals to Space-Like Separated Regions
}

\author{
Y. Aharonov ${ }^{\mathrm{a}, \mathrm{b}}$ and L. Vaidman ${ }^{\mathrm{a}, \mathrm{c}}$ \\ ${ }^{a}$ School of Physics and Astronomy, Raymond and Beverly Sackler Faculty of Exact Sciences, \\ Tel-Aviv University, Tel-Aviv 69978, Israel \\ ${ }^{b}$ Physics Department, University of South Carolina, Columbia, South Carolina 29208, USA \\ ${ }^{\mathrm{c}}$ Centre for Quantum Computation, Department of Physics, University of Oxford, \\ Clarendon Laboratory, Parks Road, Oxford OX13PU, England
}

Reprint requests to Prof. L. V.; E-mail: vaidman@post.tau.ac.il

Z. Naturforsch. 56 a, 20-26 (2001); received February 8, 2001

Presented at the 3rd Workshop on Mysteries, Puzzles and Paradoxes in Quantum Mechanics, Gargnano, Italy, September 17 - 23, 2000.

\begin{abstract}
Two recent works suggest a possibility of sending signals to a space-like separated region, contrary to the spirit of special relativity. In the first work (J. Grunhaus, S. Popescu, and D. Rohrlich, Phys. Rev. A 53, 3781 (1996)) it has been shown that sending signals to a particular union of space-like separated regions cannot cause causality paradoxes. Another work (Y. Aharonov and L. Vaidman, Phys. Rev. A 61, 052108 (2000)) showed that the relative phase of the quantum superposition of a particle at two separate locations can be measured locally. Together with the possibility of changing the relative phase in a nonlocal way using the potential effect we, apparently, have a method of sending signals to space-like separated regions. These arguments are critically analyzed in this paper.
\end{abstract}

Key words: Superluminal Signaling; Quantum Nonlocality; Quantum Paradoxes.

\section{Introduction}

Consider three space-like separated regions $A, B$ and $O$, Figure 1. Assume that we are in $O$ and we want to send a signal to $A$ and $B$. Obviously we can send the signal neither to $A$ nor to $B$. If we can, it will mean that in some Lorentz frame the signal is received before it was sent. Such a causality paradox is so robust that it has no right to be considered even in a conference on paradoxes.

But let us ask this less trivial question: is it possible to send the signal from $O$ to the union of regions $A \cup B$ ? Let us spell out what we mean by that. Can we make an operation in $O$ which will lead to an observable change in $A \cup B$ ? An observer in $A$ alone as well as an observer in $B$ alone will not be able to observe the change, but the information they both get does represent the change. Any local observer might know it only after the information from the other site will reach him, but these pieces of information are created and irreversibly recorded in $A$ and $B$.

The simplest implementation of this situation is the creation of a random bit in $A$ and another random bit

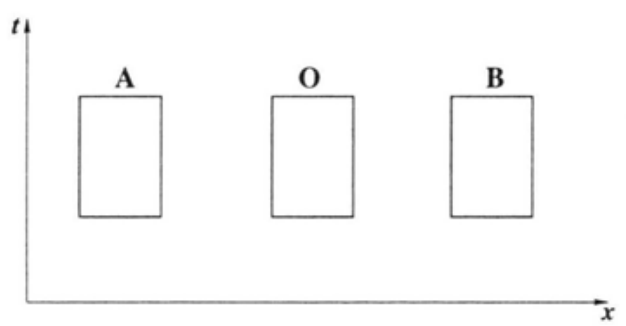

Fig. 1. A space-time diagram of space-like separated regions $A, B$, and $O$. Sending signals from $O$ to $A \cup B$ is considered.

in $B$. Since the bits are "random", changing them does not change the information each of them contains: random bits contain no information. However, these bits might be correlated or anti-correlated. The parity of these bits is the information which is stored in the union $A \cup B$. This information might exist in the union even when no information is contained in $A$ and $B$ separately.

Sending signals of this kind from $O$ to $A \cup B$ does not lead to causality paradoxes: there is no Lorentz frame in which the signal is arrived before it was sent. 
In every frame the signal has sent before some part of the information encoding the arrived signal was actualized.

Recently, Grunhaus, Popescu, and Rohrlich analyzed a similar situation [1]. They considered a "jamming of nonlocal quantum correlations". This "jamming" is exactly this kind of producing an observable change in the union of space-like separated regions. They derived a simple criterion for jamming which does not lead to causality paradoxes: the overlap of the future cones of $A$ and $B$ has to be inside the future cone of $O$, the region of the operation of the jammer. The regions $A, B$ and $O$, shown in Fig. 1, obviously fulfill this criterion.

Quantum theory does allow some kind of an instantaneous change. If we have an EPR pair of spin- $\frac{1}{2}$ particles, one located in $A$ and another located in $B$,

$$
|\Psi\rangle_{E P R}=\frac{1}{\sqrt{2}}\left(|\uparrow\rangle_{A}|\downarrow\rangle_{B}-|\downarrow\rangle_{A}|\uparrow\rangle_{B}\right),
$$

then the spin measurement in $B$ will change the state of the spin in $A$ from a mixture to a pure state. However, the information content of $A$ is not changed. The pure state which is created cannot be fixed at $B$. What is fixed is the choice between two states: one of them is created at random. Thus, by the operation at $B$, a statistical mixture is created out of the quantum mixture at $A$. But the information content of this statistical mixture is equal to the information content of the quantum mixture (it is zero, for the EPR case).

So the question is: "Can we, in the framework of quantum theory, make an observable change in the union $A \cup B$ ?" If we can, it will not lead to any causality paradox. Still, it will be somewhat paradoxical, since the spirit of special relativity tells us that there should be no "action at a distance", i. e., one cannot cause a change in a space-like separated region. The change discussed in the preceding paragraph is not so problematic. In the framework of the manyworlds interpretation there is no change at all: the statistical mixture in a particular world corresponds to the quantum mixture in the context of the quantum state of the Universe. Performing the spin measurement in $B$ on a particle from the EPR pair will not change anything about the particle in $A$ : it will remain to be in a mixed quantum state. The change in the correlation in the union $A \cup B$ we discuss here is of a different type: there is no interpretation according to which no change takes place.
Our recent work on the non-locality of a quantum wave [2] might suggest that, nevertheless, the quantum theory does allow transmission of such signals. We have found that a quantum wave of a single boson in a superposition of being in $A$ and $B$ leads to the EPR-type correlations between the two sites. These correlations are governed by the relative phase between the two parts in the superposition. But this relative phase can be changed in a non-local way through a potential effect such as the Aharonov-Bohm effect. The effect can be generated by action in a space-like separated region $O$. Therefore, it seems that one can cause an observable change in the union of space-like separated regions!

In the next section we describe the framework in which the measurements of a quantum wave are considered. Section III describes a gedanken experiment performed on a single photon. Section IV describes a proposal for a realistic experiment performed on a single photon and discusses the general structure of this method. Section V describes the application of the method for a single charged boson. Section VI analyzes the changing of the relative phase of the quantum state of the boson and, finally, in Section VII the paradox is resolved.

\section{The Framework of the Analysis}

We consider a quantum wave which is an equalweights superposition of two localized wave packets in two separate locations:

$$
|\Psi\rangle=\frac{1}{\sqrt{2}}\left(|a\rangle+e^{i \phi}|b\rangle\right) .
$$

We will analyze various simultaneous (in a particular Lorentz frame) measurements performed in these two locations; see Figure 2. We will denote by $A$ and $B$

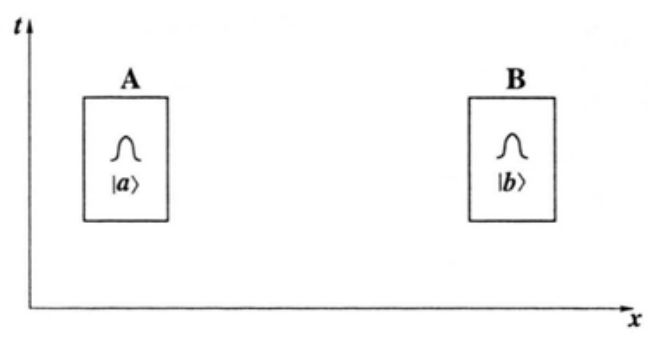

Fig. 2. Space-time diagram of the measurements performed on the quantum wave (2). 
the space-time regions of these measurements. The wave packet $|a\rangle$ is localized inside the spatial region of $A$ and the wave packet $|b\rangle$ is localized inside the spatial region of $B$.

In order to be able to make our analysis we have to specify exactly the meaning of space-time regions $A$ and $B$. Are the positions of $A$ and $B$ fixed relative to each other or are they fixed relative to an external reference frame? Are there fixed directions in $A$ and $B$ such that measuring devices can be aligned according to them? Is the time in $A$ and $B$ defined relative to local clocks, or relative to an external clock? What are the measuring devices which are available in $A$ and $B$ ? All these questions are relevant. We have to specify what is given in $A$ and $B$ prior to bringing the quantum wave there in order to distinguish effects related to the quantum wave from the effects arising from our preparation and/or definition of the sites $A$ and $B$.

We make the following assumptions:

(i) There is an external inertial frame which is massive enough so that it can be considered classical.

(ii) There is no prior entanglement of physical systems between the sites $A$ and $B$. The two laboratories in $A$ and $B$ are also massive enough so that the measurements performed on the quantum wave can be considered measurements performed with classical apparatuses. However, for various aspects of our analysis we will have to consider the two laboratories as quantum systems. We assume that relative to the external reference frame the two laboratories are initially described by a product quantum state $\left|\Psi_{A}\right\rangle\left|\Psi_{B}\right\rangle$.

(iii) There is no entanglement between the locations of the apparatuses in $A$ and the wave packet $|a\rangle$ (as well as between location of the apparatuses in $B$ and the wave packet $|b\rangle$ ). Instead, the fact that apparatus $A$ measures $|a\rangle$ and apparatus $B$ measures $|b\rangle$ is achieved via localization relative to the external frame. The measuring devices and the wave packets are well localized at the same place. This can be expressed by the equations

$$
\begin{aligned}
& \langle a|\hat{x}| a\rangle=\left\langle\hat{x}_{\mathrm{MD}_{A}}\right\rangle, \\
& \langle b|\hat{x}| b\rangle=\left\langle\hat{x}_{\mathrm{MD}_{B}}\right\rangle,
\end{aligned}
$$

where $x_{\mathrm{MD}_{A}}\left(x_{\mathrm{MD}_{B}}\right)$ are the variables that describe the location of the interaction region of the measuring devices in $A$ (in $B$ ). It is assumed that the wave packet $|a\rangle$ remains in the space region $A$ (and $|b\rangle$ remains in $B$ ) during the time of the measurements.

(iv) Measurements in $A$ and in $B$ are performed by local measuring devices activated by local clocks, say, at the internal time $\tau_{A}=\tau_{B}=0$. The clocks are well synchronized with the time $t$ of the external (classical) clock:

$$
\left\langle\tau_{A}(t)\right\rangle=\left\langle\tau_{B}(t)\right\rangle=t,
$$

and the spreads of the clock pointer variables $\Delta \tau_{A}$, $\Delta \tau_{B}$ are small during the experiment. Again, as stated in (ii), there is no entanglement between clocks in $A$ and in $B$.

The assumptions can be summarized as follows: a measurement in $A$, the space-time point relative to an external classical frame, means a measurement performed by local apparatuses in $A$ triggered by the local clock. The apparatuses and clocks in $A$ are not entangled with the apparatuses and the clocks in $B$.

\section{Single-photon Non-locality: a Gedanken Experiment}

Let us start with considering a photon in a state (2). The photon in a state (2) exhibits the non-locality of the EPR correlations. The state of the photon (2) can be written in the form

$$
|\Psi\rangle=\frac{1}{\sqrt{2}}\left(|1\rangle_{A}|0\rangle_{B}+e^{i \phi}|0\rangle_{A}|1\rangle_{B}\right),
$$

where $|1\rangle_{A} \equiv|a\rangle$ and $|1\rangle_{B} \equiv|b\rangle$. This form shows explicitly the isomorphism with the EPR state (1).

In order to get the EPR-type correlations we must be able to perform measurements on the photon analogous to the spin measurements in arbitrary direction. The analog of the spin measurement in the $\hat{z}$ direction is trivial: it is observing the presence of the photon in a particular location. A gedanken experiment yielding the analog of the spin measurements on the EPR pair in arbitrary directions is as follows [3]. Let us consider, in addition to the photon, a pair of spin- $\frac{1}{2}$ particles, one located in $A$ and one in $B$; see Figure 3. Both particles are originally in a spin "down" state in the $\hat{z}$ direction. In the locations $A$ and $B$ there are magnetic fields in the $\hat{z}$ direction such that the energy difference between the "up" and "down" states equals exactly the energy of the photon. Then we construct a physical mechanism of absorption and emission of the 


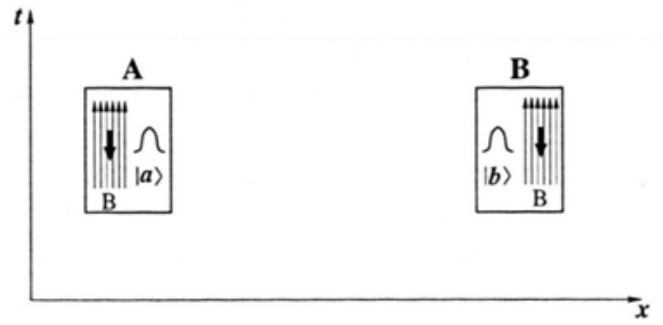

Fig. 3. Swapping of the single-photon state with the entangled state of two spin- $\frac{1}{2}$ particles.

photon by the spin which is described by the unitary transformation in each site:

$$
\begin{aligned}
|1\rangle|\downarrow\rangle & \leftrightarrow|0\rangle|\uparrow\rangle, \\
|1\rangle|\uparrow\rangle & \leftrightarrow|1\rangle|\uparrow\rangle, \\
|0\rangle|\downarrow\rangle & \leftrightarrow|0\rangle|\downarrow\rangle .
\end{aligned}
$$

This transformation swaps the quantum state of the photon and the quantum state of the pair of spin- $\frac{1}{2}$ particles as follows:

$$
\begin{gathered}
\frac{1}{\sqrt{2}}\left(|1\rangle_{A}|0\rangle_{B}+e^{i \phi}|0\rangle_{A}|1\rangle_{B}\right)|\downarrow\rangle_{A}|\downarrow\rangle_{B} \rightarrow \\
\frac{1}{\sqrt{2}}|0\rangle_{A}|0\rangle_{B}\left(|\uparrow\rangle_{A}|\downarrow\rangle_{B}+e^{i \phi}|\downarrow\rangle_{A}|\uparrow\rangle_{B}\right) .
\end{gathered}
$$

Thus, we can obtain nonlocal correlations of the EPR state starting with a single photon, swapping its state to the state of the pair of spin- $\frac{1}{2}$ particles, and then making appropriate spin component measurements. Statistical analysis of the correlations between the results of spin measurements in $A$ and in $B$ allows us to find the phase $\phi$. For example, the probabilities for coincidence and anti-coincidence in the $x$ spin measurements are given by

$$
\begin{aligned}
& \operatorname{prob}\left(\left|\uparrow_{x}\right\rangle\left|\uparrow_{x}\right\rangle\right)=\operatorname{prob}\left(\left|\downarrow_{x}\right\rangle\left|\downarrow_{x}\right\rangle\right)=\frac{1}{4}\left|1+e^{i \phi}\right|^{2}, \\
& \operatorname{prob}\left(\left|\uparrow_{x}\right\rangle\left|\downarrow_{x}\right\rangle\right)=\operatorname{prob}\left(\left|\downarrow_{x}\right\rangle\left|\uparrow_{x}\right\rangle\right)=\frac{1}{4}\left|1-e^{i \phi}\right|^{2} .
\end{aligned}
$$

We have shown that, in principal, the non-locality of a single photon is equivalent to the non-locality of the EPR pair. Now we will turn to the discussion of the possibilities of manifestation of this non-locality in real experiments and will try to explore the nature of this equivalence.

\section{Single-photon Non-locality: a Realistic Experiment}

We are not aware of experiments in which a spin in a magnetic field absorbs a photon with high efficiency. However, there is an equivalent operation which is performed in laboratories. There have been several proposals [4 - 7] how to obtain quantum correlations based on such and similar systems. Recently there has been a very significant progress in microwave cavity technology and there are experiments in which Rydberg atoms that operate as two-level systems absorb and emit photons into a microwave cavity with a very high efficiency [8]. The excited state $|e\rangle$ and the ground state $|g\rangle$ of the atom are isomorphic to the $|\uparrow\rangle$ and $|\downarrow\rangle$ states of a spin- $\frac{1}{2}$ particle. For the atom, measuring the analog of the $z$ spin component is trivial: it is the test whether the atom is in the excited state or the ground state. For measurements analogous to the spin measurements in other directions there is an experimental solution too. Using appropriate laser pulses the atom state can be "rotated" in the two dimensional Hilbert space of ground and excited states in any desired way. Thus, any two orthogonal states can be rotated to the $|e\rangle$ and $|g\rangle$ states and, then, a measurement which distinguishes between the ground and excited states distinguishes, in fact, between the original orthogonal states.

The Hamiltonian which leads to the required interactions can be written in the form

$$
H=a^{\dagger}|g\rangle\langle e|+a| e\rangle\langle g|,
$$

where $a^{\dagger}, a$ are creation and annihilation operators of the photon. This Hamiltonian is responsible for the two needed operations. First, such a coupling between the photon in the cavity in $A$ and the atom in $A$ together with a similar coupling in $B$ swaps the state (6) to the state of the two Rydberg atoms:

$$
\begin{gathered}
\frac{1}{\sqrt{2}}\left(|1\rangle_{A}|0\rangle_{B}+e^{i \phi}|0\rangle_{A}|1\rangle_{B}\right)|g\rangle_{A}|g\rangle_{B} \rightarrow \\
\frac{1}{\sqrt{2}}|0\rangle_{A}|0\rangle_{B}\left(|e\rangle_{A}|g\rangle_{B}+e^{i \phi}|g\rangle_{A}|e\rangle_{B}\right) .
\end{gathered}
$$

The same Hamiltonian can also lead to an arbitrary rotation of the atomic state. To this end the atom has 
to be coupled to a cavity with a coherent state of photons,

$$
|\alpha\rangle=e^{-\frac{|\alpha|^{2}}{2}} \sum_{n=0}^{\infty} \frac{\alpha^{n}}{\sqrt{n !}}|n\rangle .
$$

The phase of $\alpha$ specifies the axis of rotation and the absolute value of $\alpha$ specifies the rate of rotation. For example, the time evolution of an atom starting at $t=0$ in the ground state is

$$
|\Psi(t)\rangle=\cos (|\alpha| t)|g\rangle+\frac{\alpha}{i|\alpha|} \sin (|\alpha| t)|e\rangle .
$$

This is correct when we make the approximation $a^{\dagger}|\alpha\rangle \simeq \alpha^{*}|\alpha\rangle$. This approximation becomes precise in the limit of large $|\alpha|$ corresponding to a classical electromagnetic field. The Hamiltonian (11) is actually implemented in laser-aided manipulations of Rydberg atoms passing through microwave cavities.

\section{Charged-boson Non-locality}

Conceptually, the above scheme can be applied to any type of bosons (instead of photons), even charged bosons. An example of a (gedanken) Hamiltonian for this case describes a proton $|p\rangle$ which creates a neutron $|n\rangle$ by absorbing a negatively charged "meson":

$$
H=a_{m}^{\dagger}|p\rangle\left\langle n\left|+a_{m}\right| n\right\rangle\langle p|,
$$

where $a_{m}^{\dagger}, a_{m}$ are creation and annihilation operators of the meson. This Hamiltonian swaps the state of the meson (now written in the form (6)) and the state of the nucleon pair:

$$
\begin{gathered}
\frac{1}{\sqrt{2}}\left(|1\rangle_{A}|0\rangle_{B}+e^{i \phi}|0\rangle_{A}|1\rangle_{B}\right)|p\rangle_{A}|p\rangle_{B} \rightarrow \\
\frac{1}{\sqrt{2}}|0\rangle_{A}|0\rangle_{B}\left(|n\rangle_{A}|p\rangle_{B}+e^{i \phi}|p\rangle_{A}|n\rangle_{B}\right) .
\end{gathered}
$$

Since there is no direct measurement of a superposition of proton and neutron, we need again a procedure which rotates the superposition states of a nucleon to neutron or proton state. This rotation requires coherent states of mesons which would be, in this case, a coherent superposition of states with different charge. Due to strong electro-magnetic interaction the coherent state will decohere very fast. This is essentially an environmentally induced "charge super-selection rule" which prevents stable coherent superpositions of states with different charge. It is important that there is no exact charge super-selection rule which would prevent, in principle, performing the experimental scheme presented above. Indeed, Aharonov and Susskind (AS) [9] proposed a method for measuring the relative phase between states with different charge, thus showing that there is no exact charge super-selection rule. In their method one can measure the phase even if the whole system (the observed particle and the measuring device) is in an eigenstate of charge. This corresponds to initial entanglement between measuring devices in $A$ and $B$ and thus will not be suitable for the present procedure. Here we assume the existence of superpositions of different charge states: only then it is possible that the quantum state of measuring devices in $A$ and $B$ is a product state.

There are some arguments that the total charge of the universe is zero and therefore, we cannot have a product of coherent states of charged particles in $A$ and in $B$. More sophisticated analysis has to be performed: since the observable variables are only relative variables, the final conclusion will be as in the AS paper [9]: conceptually, there is no constraint on a measurement of the relative phase of a charged boson, but decoherence will prevent construction of any realistic experiment. See also very different arguments against exact super-selection rule by Giulini [10].

\section{Is it Possible to Change the Phase in a Nonlocal Way?}

Now we can come back to the original question of this paper. We have shown that the phase $\phi$ for boson state (2) is locally measurable. Given an ensemble of bosons with identical phase $\phi$ we can generate a set of numbers (results of measurements) in $A$ and another set of numbers in B, such that the two sets together yield $\phi$. Moreover, it seems that this phase can be changed non-locally via the time-dependent (scalar) $\mathrm{AB}$ effect obtained by changing the relative potential between the two parts of the wave during the time they are separated.

For a charged particle this can be achieved by moving two large oppositely charged parallel plates located between the wave packets; see Figure 4a. The two plates are placed originally one on top of the other, i.e., there is no charge distribution and, therefore, there is no electric field anywhere. The plates are then moved a distance $d$ apart and then, after 


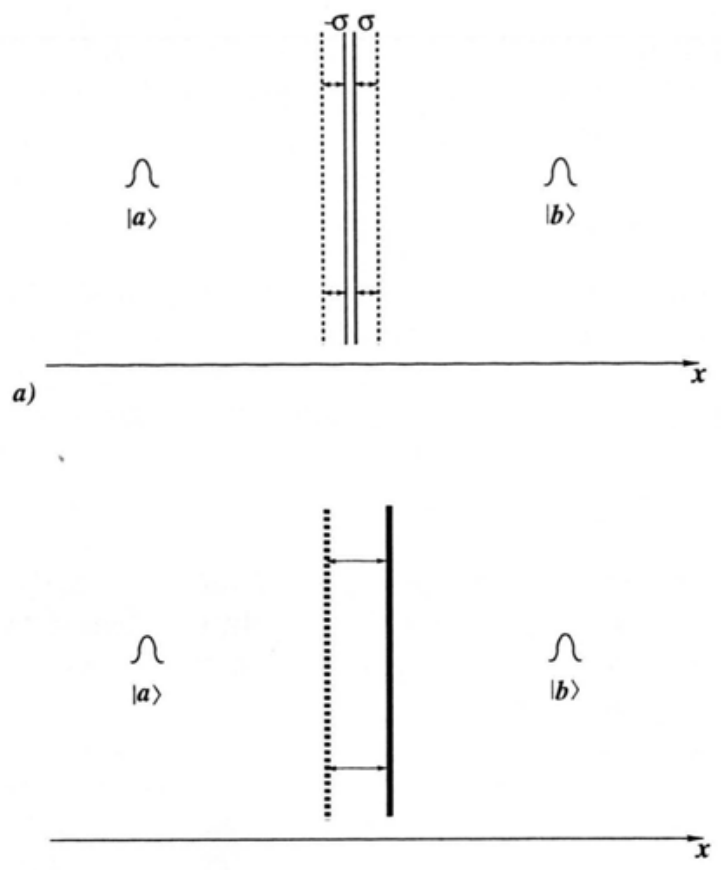

b)

Fig. 4. a) Parallel-plate condenser with charged plates, originally one on top of the other, is opened (by moving the plates apart) for a short time while the wave packets $|a\rangle$ and $|b\rangle$ are far apart. This operation introduces change in the electric potentials between the locations of $|a\rangle$ and $|b\rangle$ which generates the $\mathrm{AB}$ phase. b) A massive plate produces different gravitational potential at the locations of the wave-packets if it is closer to one of them. The relative phase is changed if the plate is moved or not moved.

a short time $t$, they are brought back. We will call such an operation "opening a condenser". Opening the condenser with the charge density $\sigma$ will lead to the change of the relative phase of the particle with charge $q$ :

$$
\Delta \phi=\frac{4 \pi \sigma d q t}{\hbar} .
$$

For $\Delta \phi=\pi$ the operation seemingly sends one bit of information from $O$ to $A \cup B$.

Consider now a neutral boson state. A massive plate in between the regions $A$ and $B$ which we move or not move towards one of the sites will introduce the phase shift in complete analogy with the scalar $\mathrm{AB}$ effect, Figure 4b. The difference here is that the gravitational fields in the regions $A$ and $B$ are not zero, but

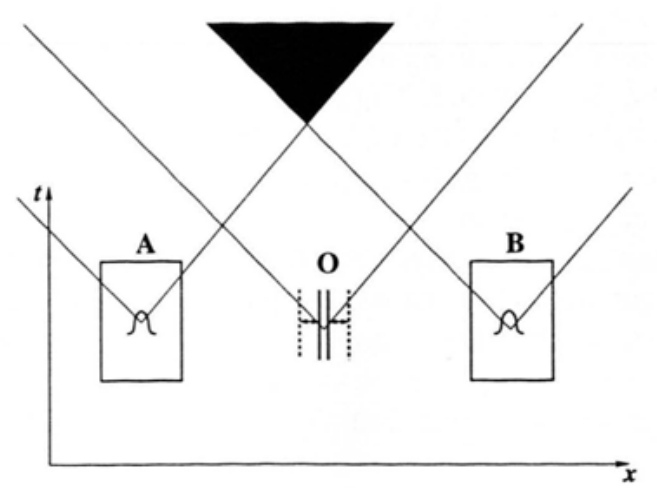

Fig. 5. Apparent sending of signals to a union of space-like separated regions. An operation in $O$, like an opening the condenser for a period of time, apparently changes the correlations between measurements in $A$ and $B$. No signal is sent from $O$, neither to $A$ nor to $B$, but the signal is sent to the union of $A$ and $B$. The intersection of light cones originated at $A$ and at $B$ lies inside the light cone originated at $O$. Therefore, the action of the condenser falls into the category of "jammers" considered in [1].

the fields are not affected by the motion of the plate. Thus, again, it seems that by an action in a localized region we can send information to a space-like separated region. Moving or not moving the massive plate changes the relative phase of the components of the quantum wave, and, therefore, apparently changes correlations in the results of measurements in $A$ and $B$; see Figure 5.

\section{Resolution of the Paradox}

In spite of the fact that we cannot reach a causality paradox using the procedure described above, it clearly contradicts the spirit, if not the letter, of special relativity. And, in fact, it is impossible. We can cause an observable change neither in a localized space-like separated region nor in the union of such regions.

It is incorrect that the opening of a condenser will change correlations between results of measurements in $A$ and $B$. It must be incorrect because we should be able to use a covariant gauge in which changes in the potentials take place only inside the light cone. However, we can explain this phenomena also in a standard (Coulomb) gauge. In our scheme the measurements in local sites include interactions with coherent states of auxiliary particles, particles which are identical to the particle in a superposition. Therefore, if the particle in question is charged, the auxiliary particles are also 
charged and opening the condenser changes the phase of the coherent state in such a way that the correlations are not changed. The gauge which we choose changes the description of auxiliary particles too, so that the probabilities for results of measurements remain gauge invariant.

Consider now a neutral boson state. The resolution of the paradox in this case is similar to the resolution of Einstein's paradox of an exact energy of an exact clock [11]. The explanation is that the pointers of the local clocks are shifted. Simultaneity between $A$ and $B$ is altered due to the action of the massive plate.

[1] J. Grunhaus, S. Popescu, and D. Rohrlich, Phys. Rev. A 53, 3781 (1996).

[2] Y. Aharonov and L. Vaidman, Phys. Rev. A 61, 052108 (2000).

[3] L. Vaidman, Phys. Rev. Lett. 75, 2063 (1995).

[4] S. M. Tan, D. F. Walls, and M. J. Collett, Phys. Rev. Lett. 66, 2521991.

[5] M. Freyberger, Phys. Rev. A 51, 3347 (1993).

[6] L. Hardy, Phys. Rev. Lett. 73, 2279 (1994).

[7] C. C. Gerry, Phys. Rev. A 53, 4583 (1996).
Since in our case local clocks activate the measurements, the shift in the pointer will lead to a change. This change compensates exactly for the phase change of the boson.

\section{Acknowledgements}

This research was supported in part by grant $471 / 98$ of the Basic Research Foundation (administered by the Israel Academy of Sciences and Humanities), the NSF grant PHY-9971005, and the EPS grant of Research Council GR/N33058.

[8] S. Haroche, M. Brune, J. R. Raimond, Philos. T. Roy. Soc. A 355 (1733) 2367 (1997).

[9] Y. Aharonov and L. Susskind, Phys. Rev. 155, 1428 (1967).

[10] D. Giulini, in "Decoherence: Theoretical, Experimental, and Conceptual Problems", P. Blanchard et al. (eds.), Lecture Notes in Physics Vol. 538, SpringerVerlag, Berlin 2000.

[11] A. Einstein, R. C. Tolman, and B. Podolsky, Phys. Rev. 37, 780 (1931). 